\title{
BRANCHING PROCESSES WITH RANDOM ENVIRONMENTS ${ }^{1}$
}

\section{BY KRISHNA B. ATHREYA AND SAMUEL KARLIN}

Communicated by David Blackwell, December 4, 1969

Let $(\Omega, F, P)$ be a probability space. Let $9 \pi$ designate the collection of all probability distributions $\left\{\bar{p}=\left\{p_{i}\right\}_{i=0}^{\infty}, p_{i} \geqq 0, \sum p_{i}=1\right\}$ on the nonnegative integers satisfying the further constraints

$$
\sum_{i=0}^{\infty} i p_{i}<\infty, \quad 0 \leqq p_{0}+p_{1}<1 .
$$

Let $\zeta_{i}(\omega) ; i=0,1,2, \cdots$ be a sequence of random mappings from $(\Omega, F, P)$ into $\left(l_{\infty}, \Theta_{\infty}\right)$ where $\Theta_{\infty}$ is the Borel $\sigma$-algebra in $l_{\infty}$ (the Banach space of bounded sequences of real numbers) generated by the product topology. We assume

$$
P\left\{\omega ; \zeta_{i}(\omega) \in \Re \quad \text { for all } i\right\}=1 .
$$

For any $\zeta \in \mathfrak{N}$ associate the p.g.f.

$$
\phi_{5}(s)=\sum_{i=0}^{\infty} p_{i}(\zeta) s^{i} \quad|s| \leqq 1 .
$$

Let $Z_{n}(\omega), n \geqq 0$ be a sequence of nonnegative integer valued random variables defined on $(\Omega, F, P)$. For any collection $D$ of random elements on $(\Omega, F, P)$ let $\sigma(D)$ denote the sub $\sigma$-algebra of $F$ generated by $D$. Set

$$
\begin{aligned}
F_{n}(\bar{\zeta}) & =\sigma\left(\zeta_{0}, \zeta_{1}, \cdots, \zeta_{n}\right), \quad F(\zeta)=\bigcup_{n \geq 1} F_{n}(\bar{\zeta}), \\
F_{n, z}(\zeta) & =\sigma\left(\zeta_{0}, \zeta_{1}, \cdots, \zeta_{n}, Z_{0}, Z_{1}, \cdots, Z_{n}\right) .
\end{aligned}
$$

We postulate that $\left\{Z_{n}\right\}$ satisfy the recurrence relations

$$
E\left(s^{Z_{n+1}} \mid F_{n, z}\right)=\left[\phi_{S_{n}}(s)\right]^{Z_{n}} \quad \text { a.s. }
$$

and for any set of integers $1 \leqq n_{1}<n_{2}<\cdots<n_{k}$ with $\left|s_{i}\right| \leqq 1$,

AMS Subject Classifications. Primary 6067; Secondary 6030.

Key Words and Phrases. Branching processes, random environments, stationary ergodic process, extinction probability, limit theorems.

${ }^{1}$ Sponsored in part by the Mathematics Research Center, United States Army, under contract No. DA-31-124-ARO-462, University of Wisconsin, Madison, Wisconsin, 53706, and in part under contract NOO14-67-A-0112-0015 at Stanford University. 
$i=1,2, \cdots, k$

(6) $E\left(s_{1}^{Z_{n_{1}}}{s_{2}}_{Z_{n_{2}}}^{Z_{n_{2}}} \ldots s_{s_{k}}^{Z_{n_{k}}} \mid F(\xi) ; Z_{0}=m\right)$

$$
=\left[E\left(s_{1}^{z_{n_{1}}} s_{s_{2}}^{Z_{n_{2}}} \ldots s_{s_{n_{k}}}^{Z_{n_{k}}} \mid F(\xi) ; Z_{0}=1\right)\right]^{m} .
$$

The proof of existence of a process satisfying these axioms is routine and parallels the constructions given in Harris [1]. The process $\left\{Z_{n}(\omega) ; n=0,1,2, \cdots\right\}$ is called a branching process with random environments (B.P.R.E.) and the process $\bar{\zeta}=\left(\zeta_{0}(\omega), \zeta_{1}(\omega), \cdots\right)$ the environmental process.

A special example of this was first introduced by Wilkinson and Smith [5] (see also [4]). They considered the case when $\bar{\xi}=\left\{\zeta_{i}(\omega)\right.$; $i=0,1,2, \ldots\}$ is a sequence of independent identically distributed random variables. Their efforts were devoted to ascertaining criteria delimiting certain extinction of the process. We study this problem under less restrictive assumptions on the environmental process $\xi$. We also deduce limit theorems for $Z_{n}$ paralleling those of the classical Galton-Watson process.

An easy consequence of (5) and (6) is

\section{LEMMA 1.}

$$
E\left(s^{Z_{n+1}} \mid Z_{0}=k, F_{n}(\bar{\zeta})\right)=\left[\phi_{\zeta_{0}}\left(\phi_{\zeta_{1}}\left(\cdots\left(\phi_{\zeta_{n}}(s)\right) \cdots\right)\right]^{k} .\right.
$$

Henceforth, unless stated explicitly otherwise we assume that $\zeta_{i}(\omega)$, $i=0,1,2, \ldots$ is a stationary ergodic process.

Let

$$
B=\left\{\omega ; Z_{n}(\omega)=0 \text { for some } n\right\}, \quad \begin{aligned}
q_{k} & =P\left(B \mid Z_{0}=k\right), \\
q_{k}(\xi) & =P\left(B \mid Z_{0}=k, F(\xi)\right) .
\end{aligned}
$$

We refer to $B$ as the set of extinction and $q_{k}, q_{k}(\bar{\zeta})$ extinction probabilities. It is clear from $(7)$ that $q_{k}(\bar{\zeta})=\left[q_{1}(\bar{\zeta})\right]^{k}$ a.s. and $q_{k}=E\left(\left[q_{1}(\bar{\zeta})\right]^{k}\right)$, thus making $\left\{q_{k}: k=1,2, \cdots\right\}$ a moment sequence, (see Smith and Wilkinson [5]).

Since the sequence of events $B_{n}=\left\{\omega: Z_{n}(\omega)=0\right\}$ increases to $B$ we have $q(\bar{\zeta})=\lim _{n \rightarrow \infty} \phi_{\zeta_{0}}\left(\phi_{\zeta_{1}}\left(\cdots\left(\phi_{\zeta_{n}}(0) \cdots\right)=E\left(\chi_{B} \mid F(\bar{\zeta})\right)\right.\right.$ where $\chi_{B}$ denotes the indicator function of the set $B$. An immediate consequence of this formula is the important functional relationship

$$
q(\bar{\zeta})=\phi_{\zeta_{0}}(q(T \bar{\zeta}))
$$

where $T$ denotes the shift transformation $T \bar{\zeta}=T\left(\zeta_{0}, \zeta_{1}, \zeta_{2}, \cdots\right)$ $=\left(\zeta_{1}, \zeta_{2}, \zeta_{3}, \cdots\right)$. Recalling the stipulations of (1) and (2) we may conclude: The sets $\{\omega: q(\bar{\zeta})=1\}$ and $\{\omega: q(T \bar{\zeta})=1\}$ coincide modulo 
a set of probability zero from which ensues the assertion $\mathrm{P}(q(\xi)=1)$ $=0$ or 1 .

We now exhibit a necessary condition for noncertain extinction i.e., for $P(q(\bar{\zeta})<1)=1$. For any real number $a$, we employ the symbols $a^{+}=\max (0, a)$ and $a^{-}=-\min (a, 0)$.

Theorem 1. Suppose

$$
P(q(\bar{\zeta})<1)=1 \text { and } E\left[\left(\log \phi_{\zeta_{0}}^{\prime}(1)\right)^{+}\right]<\infty
$$

(prime designates as usual the derivative). Then

$$
E\left|\log \phi_{5_{0}}^{\prime}(1)\right|<\infty, \quad E \log \phi_{5_{0}}^{\prime}(1)>0
$$

and

$$
E\left|\log \frac{1-q\left(\bar{\zeta}_{0}\right)}{1-q\left(T \bar{\zeta}_{0}\right)}\right|<\infty, \quad E \log \left(\frac{1-q\left(\bar{\zeta}_{0}\right)}{1-q(T \bar{\zeta})}\right)=0 .
$$

We can extract from assertion (12) for some situations an integrability property.

Theorem 2. Suppose $P(q(\bar{\zeta})<1)=1, E\left[\left(\log \phi_{5_{0}}^{\prime}(1)\right)+\right]<\infty$ and $\zeta_{i}(\omega), i=0,1,2, \cdots$ are $i . i . d$. random variables $\left(\right.$ or $\left.\zeta_{i}(\omega)\right), i \geqq 0$ form an irreducible finite Markov chain). Then $E\left(-\log \left(1-\phi_{5_{0}}(0)\right)\right)<\infty$.

The result of Theorem 2 in the independence case was achieved first by W. L. Smith [4].

The converse proposition to Theorem 1 is as follows.

Theorem 3. Suppose $E\left(-\log \left(1-\phi_{5_{0}}(0)\right)\right)<\infty$ and $E\left(\log \phi_{5_{0}}^{\prime}(1)\right)-$ $<E\left[\log \phi_{\zeta 0}^{\prime}(1)\right]+\leqq \infty$. Then $P(q(\bar{\zeta})<1)=1$.

In summary, modulo mild integrability conditions extinction is certain iff $E\left(\log \phi_{\xi_{0}}^{\prime}(1)\right) \leqq 0$.

The conclusions of Theorems 1 and 3 persist in the circumstance where $\zeta_{i}$ is not ergodic but unfolds as a positive recurrent irreducible Markov chain allowing a general state space.

It is not evident in the stationary case that

$$
P\left\{Z_{n} \rightarrow 0 \text { or } \infty\right\}=1
$$

while this relation manifestly prevails in the independence case since $\left\{Z_{n} ; n=0,1,2, \ldots\right\}$ is a Markov chain and all states other than 0 are transient. Nevertheless, (13) does hold in the stationary case. The validity of (13) is established with the aid of the following theorem.

THEOREM 4. Let $\left\{\zeta_{i}, i>0\right\}$ be a stationary ergodic process. Then

(i) $\lim _{n \rightarrow \infty} \phi_{\zeta_{0}}\left(\phi_{\zeta_{1}}\left(\cdots\left(\phi_{\zeta_{n}}(s)\right) \cdots\right)=q(\xi)\right.$ for $0 \leqq s<1$ a.s. and 
(ii) there is at most one solution $\pi(\bar{\zeta})$ of the functional equation

$$
\pi(\xi)=\phi_{\zeta_{0}}(\pi(T \bar{\zeta})) \quad \text { a.s. }
$$

satisfying $P(\pi(\bar{\zeta})<1)=1$.

It can be proved that (i) implies (13).

The B.P.R.E. will be labeled supercritical, critical, or subcritical according as $E \log \phi_{s_{0}}^{\prime}(1)>0,=0$ or $<0$ respectively. The limit theorems of the classical Galton-Watson case are generalized as follows.

SUPERCRITICAL CASE. In complete analogy with the theorem of Kesten and Stigum [3] on the supercritical Galton-Watson process we obtain

Theorem 5. Let $W_{n}=Z_{n} P_{n}^{-1}$ where for $n \geqq 1, P_{n}=\prod_{j=0}^{n-1} \phi_{\zeta_{j}}^{\prime}(1-)$ and $P_{0}=Z_{0}=1$. Then, the family $\left\{W_{n} ; F_{n, z}(\bar{\zeta}) ; n=0,1,2, \cdots\right\}$ is a nonnegative martingale and hence $\lim _{n \rightarrow \infty} W_{n}=W$ exists a.s. Suppose, in addition that

$$
E\left\{\left(\phi_{\xi_{0}}^{\prime}(1)\right)^{-1} \sum_{j=2}^{\infty} p_{\xi_{0}}(j) j \log j\right\}<\infty .
$$

Then

(i) $\lim _{n \rightarrow \infty} E\left(e^{-u W_{n}} \mid F(\bar{\zeta})\right)=\psi(u, \bar{\zeta})$ where $\psi(u, \xi)$ is the unique solution of the functional equation

$$
\psi(u, \bar{\zeta})=\phi_{\zeta_{0}}\left(\psi\left(\frac{u}{\phi_{\zeta_{0}}^{\prime}(1)}, T \xi\right)\right) \quad \text { a.s. }
$$

among those satisfying $\lim _{u \downarrow 0} u^{-1}[1-\psi(u, \bar{\xi})]=1$.

(ii) $E(W \mid F(\xi))=1$ and $P(W=0 \mid F(\bar{\zeta}))=q(\bar{\zeta})$ a.s.

The treatment of critical and subcritical cases in the context of B.P.R.E. need additional conditions on the environmental process $\left\{\zeta_{t}, t \geqq 0\right\}$.

Definition 1 . The metrically transitive stationary process $\zeta_{t}$ is said to be exchangeable if the vector random variables $\left(\zeta_{i}, \zeta_{i+1}, \cdots\right.$, $\left.\zeta_{i+n}\right)$ and $\left(\zeta_{n+i}, \zeta_{n+i-1}, \cdots, \zeta_{i}\right)$ are identically distributed for each $i \geqq 0$ and $n \geqq 0$.

When $\zeta_{t}, t \geqq 0$ consists of i.i.d. random variables then $\zeta_{t}$ is manifestly an exchangeable process. Another example occurs where $\left(\zeta_{t}, t \geqq 0\right)$ is a stationary reversible ergodic Markov chain.

The following is the analogue of Yaglom's theorem for subcritical Galton-Watson processes (see [3]).

Theorem 6. Let $\left(\zeta_{t}, t \geqq 0\right)$ be an exchangeable process as in Definition 
1. Suppose $E\left|\log \phi_{5_{0}}^{\prime}(1)\right|<\infty$. In the subcritical B.P.R.E., i.e., where $E\left(\log \phi_{s_{0}}^{\prime}(1)\right)<0$ holds, there exists a p.g.f. $G(s, \xi)$ for a.e. $\xi$ such that

$$
E\left(s^{z_{n}} \mid Z_{n} \neq 0, F(\zeta)\right) \stackrel{d}{\rightarrow} G(s, \zeta)
$$

as $n \rightarrow \infty$ where $d$ means convergence in distribution. In the critical case, that is where $E\left(\log \phi_{\zeta_{0}}^{\prime}(1)\right)=0$, (15) persists but $G(s, \bar{\zeta}) \equiv 0$ a.s.

The subcritical Galton-Watson process is characterized in that the mean number of progeny per parent is less than 1 . The analog of this property in the B.P.R.E. case is the content of the following theorem.

Theorem 7. Let $\zeta_{t}, t \geqq 0$ be an exchangeable process. Suppose $E \mid-\log \left(1-\phi_{5_{0}}(0) \mid\right.$ and $E\left|\log \phi_{5_{0}}^{\prime}(1)\right|$ are finite. Then

$$
m(\xi)=\lim _{n \rightarrow \infty} \frac{1-\phi_{\zeta_{n}}\left(\phi_{\zeta_{n-1}}\left(\cdots\left(\phi_{\zeta_{0}}(0)\right)\right)\right)}{1-\phi_{\zeta_{n}}\left(\phi_{\zeta_{n-1}}\left(\cdots\left(\phi_{\zeta_{1}}(0)\right)\right)\right)}
$$

exists and is $\leqq 1$ a.s. The B.P.R.E. is subcritical iff $P(\xi ; m(\xi)<1)>0$.

REMARK. Notice for the special case $\phi_{5_{0}}(s)=\phi(s)$ a.s. that $m(\xi)=$ $\phi^{\prime}(1)$.

In the subcritical case we have determined (subject to a mild moment condition) the exact rate of approach to zero of $1-\phi_{5 n}\left(\phi_{5 n-1}\left(\cdots\left(\phi_{50}(s) \cdots\right)\right)\right)$ which is the generalization of geometric ergodicity in the classical case.

TheOREM 8. Assume $E\left(\log \phi_{5_{0}}^{\prime}(1)\right)^{+}<\infty, \quad E\left(\log \phi_{5_{0}}^{\prime}(1)\right)->$ $E\left(\log \phi_{50}^{\prime}(1)\right)+$ and that (14) holds. Then a.s.

$$
\lim _{n \rightarrow \infty} \frac{1-\phi_{\zeta_{n}}\left(\cdots\left(\phi_{\zeta_{0}}(s)\right) \cdots\right)}{\prod_{-0}^{n} \phi_{\xi_{i}}(1-)}=A(s, \bar{\zeta})
$$

exists and $A(s, \xi)>0$ for $0 \leqq s<1$.

We finish with a result on the extinction probability problem for the multi-type (say $p$-types) B.P.R.E. model. To review quickly the formulation we have associated with each $\xi$ a $p$-vector p.g.f. $\phi_{5}(s)$ $=\left(\phi_{\zeta}^{(1)}(s), \phi_{\zeta}^{(2)}(s), \cdots, \phi_{\xi}^{(p)}(s)\right)$. Let $A_{\zeta}=\left\|\partial \phi_{\zeta}^{(i)}(1) / \partial s_{j}\right\|$. We assume $E\left|\log \left\|A_{\xi}\right\|\right|<\infty$ where $\|A\|=\max _{i} \sum_{j=1}^{v}\left|a_{i j}\right|$. It was proved by Furstenburg and Kesten [2] that $\lim _{n \rightarrow \infty}(1 / n) \log \left\|A_{\zeta_{n}} \cdots A_{\zeta_{0}}\right\|=\pi$ exists w.p.1, $\pi<\infty$ and also $\lim _{n \rightarrow \infty}(1 / n) E\left\|\log A_{\zeta_{n}} \cdots A_{\xi_{0}}\right\|=\pi$. Clearly, for the one type process $\pi=E \log \phi_{50}^{\prime}(1)$.

Criteria for extinction or nonextinction is the substance of the following theorem. 
THEOREM 9. Let $\left(\zeta_{t}, t \geqq 0\right)$ be a process of i.i.d. random variables and let $\phi_{5}(\mathrm{~s})$ be a p-vector p.g.f. Further assume

(i) $E\left(-\log \left(1-\phi_{5_{0}}(0) \cdot v\right)\right)<\infty$ for every vector $v \gg 0$,

(ii) there exists $0<k<K<\infty$ such that

$$
\begin{aligned}
P\left[\zeta_{0} ;\left.\sup _{j, l, m} \frac{\partial^{2} \phi_{s_{0}}^{(j)}}{\partial s_{l} \partial s_{m}}\right|_{s=1} \leqq K, \quad k\right. & \leqq\left.\inf _{j, l} \frac{\partial \phi_{s_{0}}^{(j)}}{\partial s_{l}}\right|_{s=1} \\
& \left.\leqq\left.\sup _{j, l} \frac{\partial \phi_{S_{0}}^{(j)}}{\partial s_{l}}\right|_{s=1} \leqq K\right]=1 .
\end{aligned}
$$

Let $q(\bar{\zeta})=\lim _{n \rightarrow \infty} \phi_{\zeta_{0}}\left(\phi_{\zeta_{1}}, \cdots, \phi_{\zeta_{n}}(0), \cdots\right)$ denote the extinction probability vector. Then if $\pi<0 P(\boldsymbol{q}(\bar{\zeta})=1)=1$ and if $\pi>0$ then $P(q(\zeta) \ll 1)=1$. (The notation $x \ll y$ for vectors signifies that every component of $y-x$ is positive.)

Proofs and elaboration of the above results and others will appear elsewhere.

\section{REFERENCES}

1. T. E. Harris, The theory of branching processes, Chapts. 2 and 6, Die Grundlehren der math. Wissenschaften, Band 119, Springer-Verlag, Berlin and New York and Prentice-Hall, Englewood Cliffs, N.J., 1963. MR $29 \# 664$.

2. H. Furstenberg and H. Kesten, Products of random matrices, Ann. Math. Statist. 31 (1960), 457-469. MR $22 \# 12558$.

3. H. Kesten and B. P. Stigum, A limit theorem for multidimensional GaltonWatson processes, Ann. Math. Statist. 37 (1966), 1211-1223. MR 33 \#6707.

4. W. Smith, Necessary conditions for almost sure extinction of a branching process with random environment, Ann. Math. Statist. 39 (1968), 2136-2140. MR 38 \#5299.

5. W. Smith and W. Wilkinson, On branching processes in random environments, Ann Math. Statist. 40 (1969), 814-827.

University of Wisconsin, Madison, Wisconsin 53706

Stanford University, Stanford, CaLIfornia 94305 